\section{ISG-012 COST-EFFECTIVENESS OF AZACITIDINE IN THE REAL- WORLD: ANALYSIS IN HIGH-RISK PATIENTS WITH MYELODYSPLASTIC SYNDROMES FROM THE PERSPECTIVE OF A EUROPEAN PUBLIC HOSPITAL}

${ }^{1} \mathrm{~T}$ Torrecilla*, 'M Jimenez-Heredia, ${ }^{1} \mathrm{P}$ Calpe, ${ }^{2} \mathrm{M}$ Tormo. ${ }^{1}$ Hospital Clínico de Valencia, Pharmacy, Valencia, Spain; ${ }^{2}$ Hospital Clínico de Valencia, Haematology, Valencia, Spain

10.1136/ejhpharm-2019-eahpconf.12

Background Azacitidine is the recommended treatment for higher-risk myelodysplastic syndromes (MDS) in patients who are not candidates for haematopoietic transplantation. It is also used in low-risk MDSs where supportive treatment fails.

Despite its widespread use, there are no pharmacoeconomic data of azacitidine based in the real-world setting, outside the context of clinical trials.

Purpose To evaluate the incremental cost-effectiveness ratio of azacitidine versus supportive care in patients with MDS treated in a public hospital, from the payers' perspective.

Material and methods Observational retrospective study: two cohorts of patients with MDS ( $n=53$ patients each one), with similar demographic, clinical, biological and haematological characteristics. Medication consumption, transfusion support and hospital resources were accounted for each patient, according to the Valencian Community Fees Law (2016 fiscal year), and to the 2017 final hospital sale price for medicines. Overall survival since diagnosis was the measure of effectiveness.

Mean based cost-effectiveness ratio was estimated with the bootstrapping resampling technique. The average cost was calculated with the Bang-Tsiatis reweigted estimator and restricted mean survival time (RMST) was used for effectiveness. $^{1}$

Patients were stratified according to the International Prognostic Score System for risk: 25 high-risk/intermediate-2 treated with azacitidine, and 21 with supportive care.

Results Patients treated with azacitidine showed improved survival in high-risk/intermediate-2 patients (RMST: 33 versus 19 months; Kaplan-Meier median survival: 13 versus 6 months).

The mean-based cost-effectiveness ratio was $€ 16812$ per life-year gained. According to the cost-effectiveness plane, $91 \%$ of values lie in the northeast quadrant, where increased survival is achieved at increased cost. Sixty-eight per cent of the values are within the threshold $(€ 30000$ per life-year gained) of willingness to pay commonly accepted for cost effectiveness in Spain.

Conclusion Azacitidine shows a favourable cost-effectiveness ratio in high-risk intermediate- 2 patients, although with the uncertainty derived from the small sample size.

This result corroborates what is reflected in the bibliography for azacitidine cost-effectiveness, but is based on data obtained from the usual healthcare practice. On the contrary, azacitidine cost-effectiveness publications are usually based on mathematical models and data from clinical trials, which shows more favourable results than real-world practice.

\section{REFERENCE AND/OR ACKNOWLEDGEMENTS}

1. Bang $H$, Tsiatis AA. Estimating medical costs with censored data. Biometrika 2000;87;329-43. https://doi.org/10.1093/biomet/87.2.329

No conflict of interest.

\section{ISG-013 BUDGET IMPACT ANALYSIS OF LUNG CANCER IMMUNOTHERAPY: A HOSPITAL PERSPECTIVE}

E Pierobon, F Capano*, D lelo. A.O.U San Luigi Gonzaga, Pharmacy, Turin, Italy

\subsection{6/ejhpharm-2019-eahpconf.13}

Background The evaluation of the economic impact of therapies has become mandatory to guarantee sustainability to the health service, since neoplasms have assumed considerable economic burden in developed countries: $42 \%$ of the expenditure for high-cost therapies is for the most common pathologies, including lung.

Purpose To estimate a hypothesis of future expenditure for the immunotherapy in second-line treatment of non-small cell lung cancer (NSCLC), through a budget impact analysis.

Material and methods The analysis was carried out adopting a time horizon of 13 months, the hospital's perspective and considering only direct costs of drugs' purchase. The population corresponds to patients with a diagnosis of NSCLC, eligible for second-line immunotherapy.

The model used was defined based on real clinical data: patients actually treated were selected from August 2017 to August 2018, a period in which both nivolumab and pembrolizumab were available in the hospital.

The annual cost of therapy was calculated based on the milligrams of drug consumed, (thus considering the drop-in and drop-out rate) and the price of the drug according to the regional public tender. To calculate the drop-in and drop-out rate for atezolizumab, reference was made on overall survival data of the pivotal trial.

Therefore, three theoretical scenarios were considered: without introduction into therapy of atezolizumab (first); all naive patients received atezolizumab (second); and 50\% of the naive received pembrolizumab and $50 \%$ atezolizumab (third).

Results In the first scenario, the initial distribution of patients treated with pembrolizumab compared to nivolumab is 3 vs 17 (month 1), to reach 28 vs 24 (month 13), and the consequential cumulative costs are $(€ 1,131,240$ and $€ 1,481,196$ respectively), for a total of $€ 2,612,435$.

In the second scenario the cumulative costs are $€ 272526$ for pembrolizumab, $€ 623831$ for nivolumab and $€ 1,692,250$ for atezolizumab, (total of $€ 2,588,607$, $+0.9 \%$ compared with the first), while in the third scenario the costs are $€ 1,804,842$, $€ 623831$ and $€ 850406$ respectively for a total of $€ 3,279,079$ ( $+20 \%$ compared to the second).

Conclusion Based on our setting, costs are comparable in the three scenarios even if the cost per administration is almost double for pembrolizumab compared with atezolizumab. One main limitation of the study is that, in the near future, new indications and new therapies may be approved.

\section{REFERENCES AND/OR ACKNOWLEDGEMENTS}

Conflict of interest.

Corporate-sponsored research or other substantive relationships; advisory board with grant for novartis, sanofy, maya idee; and telephone interview with honoraria for doxafarma, participation in courses and conferences sponsored by various pharmaceutical companies. 